\title{
Renormalization group theory of earthquakes
}

\author{
Hubert Saleur $^{1^{*}}$, Charles G. Sammis ${ }^{2}$ and Didier Sornette ${ }^{3}$ \\ ${ }^{1}$ Department of Physics and Astronomy, University of Southem California, Los Angeles, CA 90089-0740, USA \\ "Packard Fellow \\ ${ }^{2}$ Department of Earth Sciences, University of Southern California, Los Angeles, CA 90089-0740, USA \\ ${ }^{3}$ Laboratoire de Physique de la Matière Condensée, CNRS URA190, Université des Sciences, B.P. 70, Parc Valrose, \\ 06108 Nice Cedex 2, France
}

Received 5 April 1995 - Accepted 19 October 1995 - Communicated by A. Provenzale

\begin{abstract}
We study theoretically the physical origin of the proposed discrete scale invariance of earthquake processes, at the origin of the universal log-periodic corrections to scaling, recently discovered in regional seismic activity (Sornette and Sammis (1995)). The discrete scaling symmetries which may be present at smaller scales are shown to be robust on a global scale with respect to disorder. Furthermore, a single complex exponent is sufficient in practice to capture the essential properties of the leading correction to scaling, whose real part may be renormalized by disorder, and thus be specific to the system. We then propose a new mechanism for discrete scale invariance, based on the interplay between dynamics and disorder. The existence of non-linear corrections to the renormalization group flow implies that an earthquake is not an isolated "critical point", but is accompanied by an embedded set of "critical points", its foreshocks and any subsequent shocks for which it may be a foreshock.
\end{abstract}

\section{Introduction}

A number of authors have proposed earthquake source theories based on the concept of critical points and using the Renormalization Group (Chelidze (1982); Allègre et al. (1982); Smalley et al. (1985); Sornette and Sornette (1990); Tumarkin and Shnirman (1992); Newman et al. (1993)). The usefulness of the renormalization group is based on the scale invariance of the physical processes associated with earthquakes (Toulouse and Pfeuty (1975); Wilson (1979)). This scale invariance may be either continuous or discrete (a discrete scale invariance means that only discrete rescalings are allowed). The former case corresponds to standard thermodynamic critical points (Toulouse and Pfeuty (1975); Amit (1984)) and ensures a wealth of well-behaved properties, such as the relevance of a single correlation length and the fact that critical exponents are real numbers
(Wallace and Zia (1975)). In the discrete case, new properties are expected, such as multiple correlation lengths and complex critical exponents (Jona-Lasinio (1975); Nauenberg (1975); Niemeijer and van Leeuwen (1976); [Bessis et al. (1983)). All models viewing earthquakes as critical points fall into this latter class, since they essentially assume an underlying discrete hierarchical geometrical structure (Keiis-Borok (1990)).

In (Sornette and Sammis (1995)), it has been shown that this assumption of a discrete scale invariance has a simple but potentially very important practical consequence, namely the existence of "universal" log-periodic corrections to scaling which had been previously overlooked. The corresponding specific mathematical structure of the solution of the renormalization group can account for many observed precursory seismic phenomena, such as extended quiescence and intermediate term regional foreshocks, and it suggests a new approach to earthquake prediction. Since the existence of such corrections to scaling rely fundamentally on the assumed discrete scale invariance, an assumption shared by many authors, it is important to explore its physical origin. The simplest origin of discrete scale invariance is the multi-scale geometrical organization of the crust (KeiisBorok (1990); Scholz (1990)). However, in contrast to the geometrical structure underlying the simple models developed for instance by (Chelidze (1982); Allègre et al. (1982); Smalley et al. (1985); Tumarkin and Shnirman (1992); Newman et al. (1993)), the hierarchical organization of the earth crust contains significant irregularity and disorder. It is not a priori clear that this disorder will not destroy, on larger scales, the discrete scale symmetries which may be present at smaller scales. Here, we address this question theoretically and explore the generality and robustness with respect to disorder of the universal log-periodic corrections to scaling which derive from the discrete scale invariance. Furthermore, we show quite generally that a single complex exponent is sufficient in practice to capture the essential properties 
of the leading log-periodic correction to scaling, whose real part may be renormalized by disorder, and thus be specific to the system. The universal existence of such corrections to scaling is thus not in contradiction with the specificity of their structure, thus providing potentially useful genuine fingerprints of a given system.

Is the existence of an underlying hierarchical geometrical structure the only mechanism to generate discrete scale invariance? We suggest that the answer is negative and propose a new mechanism for discrete scale invariance, based on the interplay between dynamics and disorder. In other words, this means that a suitable diffusion-like process occurring within a system characterized by uncorrelated disorder creates characteristic times (and spatial scales) spontaneously, which follow approximately a geometrical series, hence the discrete scale invariance. This idea parallels the demonstration of a spontaneous formation of fractal fault structures by repeated earthquakes in an elastic plate with uncorrelated disorder (Cowie et al. (1993); Miltenberger et al. (1993); Sornette et al. (1994)). These fractal faults turn out to be optimal structures of the disorder in a certain sense, which shows that even an uncorrelated heterogeneity contains the seed for large scale self-similar organization in the presence of a suitable dynamics.

Finally, we briefly outline how the renormalization group formalism, used beyond the linear approximation, provides a global description on a same footing, not only of a single large earthquake modelled as a critical point, but also of the set of its precursors and following earthquakes for which it may be a foreshock.

\section{Renormalization group formalism of discrete scale invariance in random systems}

\subsection{Ordered case}

The renormalization group (RG) formalism amounts basically to a decomposition of the general problem of finding the behavior of a large number of interacting elements into a succession of simple problems with a smaller number of elements, possessing effective properties which vary with the scale of observation (Toulouse and Pfeuty (1975); Wilson (1979)). We shall start from the formalism used in (Sornette and Sammis (1995)) and write a $R G$ recursion relation expressing how a change of scale with a finite scaling factor induces a change of effective time distance to the time $t_{f}$ of the large event (defined henceforth as the critical time of global (regional) failure) and a scaling of the physical observable, taken here as being the seismic energy $\epsilon$. Posing

$$
x=t_{f}-t
$$

and

$$
F(x) \equiv \epsilon
$$

such that $F$ is singular at the leading critical point $x=$ 0 , the most general discrete RG equation, for the case of a single relevant control parameter $x$, reads

$$
\begin{gathered}
x^{\prime}=\phi(x), \\
F(x)=g(x)+\frac{1}{\mu} F(\phi(x)),
\end{gathered}
$$

The function $\phi$ is called the RG flow map, and $\mu$ is a constant describing the scaling of the seismic release rate upon the discrete time rescaling (1).

The function $g(x)$ represents the non-singular part of the function $F(x)$. We assume as usual that the function $F(x)$ is continuous and that $\phi(x)$ is differentiable. Let us recall that the critical point(s) is (are) described mathematically as the time(s) at which $F(x)$ becomes singular, i.e. when there exists a finite $k$-th derivative $\frac{d^{k} F(x)}{d x^{k}}$ which becomes infinite at the singular point(s). Close to such a singularity, $\mathrm{F}(\mathrm{x})$ can be expanded as a powerlaw whose (critical) exponent provides the scaling law for the physical observable.

The formal solution of eq.(2) is obtained by considering the following definitions:

$$
f_{0}(x) \equiv g(x),
$$

and

$$
f_{n+1}(x)=g(x)+\frac{1}{\mu} f_{n}[\phi(x)], \quad n=0,1,2, \ldots
$$

It is easy to show (by induction) that

$$
f_{n}(x)=\sum_{i=0}^{n} \frac{1}{\mu^{i}} g\left[\phi^{(i)}(x)\right], \quad n>0 .
$$

Here, we have used superscripts in the form ' $(n)$ ' to designate composition, i.e.

$$
\begin{gathered}
\phi^{(2)}(x)=\phi[\phi(x)] ; \\
\phi^{(3)}(x)=\phi\left[\phi^{(2)}(x)\right] ;
\end{gathered}
$$

etc. It naturally follows that

$$
\lim _{n \rightarrow \infty} f_{n}(x)=F(x),
$$

i.e.

$$
F(x)=\sum_{i=0}^{\infty} \frac{1}{\mu^{i}} g\left[\phi^{(i)}(x)\right] .
$$

Note that the power of the RG analysis is to reconstruct the nature of the critical singularities from the embedding of scales, i.e. from the knowledge of the non-singular part $g(x)$ of the observable and the flow map $\phi(x)$ describing the effect of the change of scale on the control parameter (here the time to rupture). The connection between this formalism and the critical point 
problem stems from the fact that the critical points correspond to the unstable fixed points of the RG flow $\phi(x)$. Indeed, as in standard phase transitions, a singular behavior emerges from the infinite sum of analytic terms, describing the solution for the observable $F(x)$, if the absolute value of the eigenvalue $\lambda$ defined by

$$
\lambda=\left.\frac{d \phi}{d x}\right|_{x=\phi(x)}
$$

becomes larger than 1 , in other words, if the mapping $\phi$ becomes unstable by iteration at the corresponding (critical) fixed point (the fixed point condition ensuring that it is the same number appearing in the argument of $g($.$) in the series). In this case, the i$ th term in the series for the $k$ th derivative of $F(x)$ at the fixed point will be proportional to

$$
\left(\frac{\lambda^{k}}{\mu}\right)^{i}
$$

giving rise to a divergence for sufficiently large $k$, hence the singular behavior.

Thus, the qualitative behavior of the critical points and the corresponding critical exponents can be simply deduced from the structure of the RG flow $\phi(x)$. If $x=0$ denotes a fixed point $(\phi(0)=0)$ and $\phi(x)=\lambda x+\ldots$ is the corresponding linearized transformation, then the solution of eq.(2) close to $x=0$ is given by

$$
F(x) \sim x^{m},
$$

with

$$
\frac{\lambda^{m}}{\mu}=1
$$

yielding the real critical exponent

$$
m=\frac{\log \mu}{\log \lambda} .
$$

However, one has the identity $1=e^{i n 2 \pi}$ with $n$ being an integer, showing that a series of solutions appear with complex exponents

$$
m_{n}=m+i n \Omega,
$$

where

$$
\Omega=\frac{2 \pi}{\log \lambda}
$$

Since

$$
\operatorname{Re}\left[x^{m+i n \Omega}\right]=x^{m} \cos (n \Omega \log x)
$$

physically, the complex critical exponents describe oscillations decorating the asymptotic powerlaw $F(x) \sim x^{m}$. It is important to stress again that these Log-periodic correction to scaling stem from the discreteness of the RG equations (1) and (2), i.e. one goes from a time $t$ to another time $t^{\prime}$ which is obtained by a finite (and not an infinitely small) rescaling of $t$. This is intended to capture the existence of a discrete scaling invariance in the system.

In full generality, the previous discussion shows that the general solution to the RG equations consists in a set of complex critical exponents, corresponding to the different term in the Fourier expansion of the most general solution. We now present a formalim which shows that only the first complex exponent $n=1$ needs to be taken into account in practice, thus justifying the fitting procedure used in (Sornette and Sammis (1995)). Let us introduce the Mellin transform

$$
\hat{F}(s)=\int_{0}^{\infty} F(x) x^{s-1} d x .
$$

Transforming expression (3) in the linear approximation yields

$$
\hat{F}(s)=\hat{g}(s) \frac{\mu \lambda^{s}}{\mu \lambda^{s}-1},
$$

where $\hat{g}(s)$ is the Mellin transform of $g(x)$. Then $F(x)$ follows from the inverse Mellin transform

$$
F(x)=\int_{c-i \infty}^{c+i \infty} \hat{F}(s) x^{-s} d s
$$

which shows, by the Cauchy theorem, that $F(x)$ is the sum of terms $x^{-s_{\text {pale }}}$ with amplitude given by the residues

$$
\frac{\hat{g}\left(s_{p o l e}\right)}{\log \lambda}
$$

where $s_{\text {pole }}$ satisfies

$$
\mu \lambda^{s \text { pole }}=1 \text {, }
$$

whose solutions have already been given above $\left(s_{\text {pole }}=\right.$ $\left.-m_{n}\right)$.

In order to make further progress, one has to specify $g(x)$. We assume the general form

$$
g(x)=\log \left(\sum_{p=0}^{N} a_{p} x^{p}\right),
$$

which corresponds typically to the form of the free energy of a cluster of interacting systems. Writing

$$
\sum_{p=0}^{N} a_{p} x^{p}=\prod_{j}\left(\left(x-x_{j}\right)^{2}+y_{j}^{2}\right)
$$

since all roots should be complex, the Mellin transform of $g(x)$ involves the sum of the Mellin transform of terms

$$
\log \left(\left(x-x_{j}\right)^{2}+y_{j}^{2}\right) \sim \log \left(1+2 x \cos l+x^{2}\right),
$$

with $t$ real, i.e.

$$
\int_{0}^{\infty} d x x^{s-1} \log \left(1+2 x \cos t+x^{2}\right)=\frac{2 \pi}{s} \frac{\cos s t}{\sin \pi s}
$$


for $|t|<\pi$ and $-1<\operatorname{Re}(s)<0$. The amplitude of a given pole

$$
s_{\text {pole }}=-m+i n \Omega
$$

is thus proportional to

$$
\left|\frac{1}{m-i n \Omega} \frac{\cos (m-i n \Omega) t}{\sin (m-i n \Omega) \pi}\right| \sim e^{-n \Omega(\pi-t)} .
$$

The amplitude of the second correction $(n=2)$ compared to the first one $(n=1)$ is thus of order

$$
e^{\frac{2 \pi^{2}}{\log \lambda}\left(1-\frac{1}{\pi}\right)}
$$

For $\lambda \simeq 3$ (Sornette and Sammis (1995)) and for a typical value $t \sim 1$, this is of order $10^{-2}$. The decay is much slower (as $n^{-1}$ ) only when $t$ is very close to $\pi$, but this case should be exceptional. This thus justifies using only the first correction to scaling corresponding to

$$
m_{1}=m+i \Omega,
$$

and neglect higher terms $n>1$, as done in (Sornette and Sammis (1995)). Of course the linear approximation becomes invalid for large values of the index $i$ in the series (3), so the previous discussion must be taken with same caution. However numerical studies indicate that its main result is qualitatively correct ([Derrida et al. (1983)).

\subsection{Random systems}

We now analyze the robustness of this above RG analysis with respect to disorder in the discrete scale invariance. We consider the simplest model with disorder which consists in replacing

$$
\frac{1}{\mu^{i}} g\left(\phi^{(i)}(x)\right) \simeq \frac{1}{\mu^{i}} g\left(\lambda^{i} x\right)
$$

in eq.(3) by

$$
\frac{1}{\prod_{j=1}^{i} \mu_{j}} g\left(\left(\prod_{j=1}^{i} \lambda_{j}\right) x\right)
$$

The $\mu_{j}$ 's and $\lambda_{j}$ 's are random numbers describing the fuctuations of the scaling and of the flow map at each iteration. Taking such a random flow map at each iteration corresponds to changing the scaling factor of the RG decimation procedure, and therefore to describing a hierarchical system with disorder on the scale factor from one level to the next of the hierarchical structure. Note that this is different from the case of a hierarchical system, such as the Bethe lattice, characterized by a single scaling factor but on which each bond is carrying a different random interaction. The sum in eq.(3) is then replaced by

$$
\frac{\hat{F}(s)}{\hat{g}(s)}=\left[1+X_{1}+X_{1} X_{2}+X_{1} X_{2} X_{3}+\ldots\right],
$$

where

$$
X_{i}=\frac{1}{\mu_{i} \lambda_{i}^{s}}
$$

Noting

$$
S_{n}=\left[1+X_{n}+X_{n} X_{n+1}+X_{n} X_{n+1} X_{n+2}+\ldots\right],
$$

one has

$$
\frac{\hat{F}(s)}{\hat{g}(s)} \equiv S_{1}=1+X_{1} S_{2} .
$$

Since the random variables $X_{n}$ are independently and identically distributed,

$$
<S_{1}>=<S_{2}>
$$

where $\left\langle S_{n}\right\rangle$ is the ensemble average of $S_{n}$ over many different random realizations. Since $X_{1}$ is independent of $S_{2}$, we get

$$
<S_{1}>=\frac{1}{1-<X_{1}>}
$$

i.e.

$$
\hat{F}(s)=\hat{g}(s) \frac{1}{1-\left\langle\frac{\lambda^{-s}}{\mu}\right\rangle} .
$$

The critical exponents $s$ are given by the equation

$$
<\frac{\lambda^{-s}}{\mu}>=1 \text {. }
$$

Note that we are looking for solutions $s$ with a negative real part since the physical observable $\epsilon$ does not diverge at the critical point, only its derivative i.e. seismic rate diverge mathematically (in reality, this divergence is rounded off at some finite level due to the finite size of the earthquake). Thus, the average is dominated by the large, even if rare, values of $\lambda$.

The first consequence one can draw from this analysis is that disorder suppresses the harmonics $s_{n}=-m_{n}$ found in the ordered case, since each different power $s$ gives a different weight in the ensemble average, ensuring for instance that

$$
<\lambda^{m+i n \Omega}>\neq<\lambda^{m}><\lambda^{i \Omega}>^{n} .
$$

The fast decay of high harmonics found in the ordered case is not modified by the disorder. There is another interesting consequence of the disorder that we now analyze. Let us assume for the sake of simplicity that the disorder is only on the $\lambda$ 's. Consider the two leading terms in the solution $F(x)$. They corresponds to a first leading powerlaw with real exponent $m$ and a second log-periodic term with exponent $m^{\prime}+i \Omega$. The two exponents are such that

$$
<\lambda^{m}>=<\lambda^{m^{\prime}+i \Omega}>
$$

since they both satisfy the pole equation

$$
<\lambda^{-s}>=\mu
$$


(assuming here for the sake of pedagogy that $\mu$ is not random). Let us call $p(\lambda)$ the distribution of $\lambda$. Then,

$$
\operatorname{Re}\left(\left\langle\lambda^{m^{\prime}+i \Omega}\right\rangle\right)=\int_{0}^{\infty} d \lambda p(\lambda) \lambda^{m^{\prime}} \cos (\Omega \log \lambda) .
$$

Its modulus is less than

$$
\int_{0}^{\infty} d \lambda p(\lambda) \lambda^{m^{\prime}}
$$

and from the above identity

$$
<\lambda^{m}>=<\lambda^{m^{\prime}+i \Omega}>
$$

this implies

$$
\begin{cases}m<m^{\prime} & \text { if } \frac{d<\lambda^{m^{\prime}}}{d m^{\prime}}>0 \\ m>m^{\prime} & \text { in the reverse case }\end{cases}
$$

Therefore, depending on the specific form of the distribution $p(\lambda)$ of the RG flow map eigenvalues $\lambda$, the disorder leads to a renormalization of the real part of the critical exponent.

As a first illustration, let us assume that $p(\lambda)$ is $\log$ normal. An explicit calculation shows that $m^{\prime}>m$ in this case. The reason for this is clear : a log-normal distribution has a long tail towards large values and thus the average is dominated by the large $\lambda$ 's, all the more so when $m^{\prime}$ gets greater. This is a case where the average is an increasing function of $m^{\prime}$, hence the result $m<m^{\prime}$. The concrete consequence for earthquakes, and other relevant systems, is that the mathematical expression used in fitting seismic activity (Sornette and Sammis (1995)) should be replaced by

$\left.\epsilon(t)=A+B\left(t_{f}-t\right)^{m}+C\left(t_{f}-t\right)^{m^{\prime}} \cos \left(2 \pi \frac{\log \left(t_{f}-t\right)}{\log \lambda}+\Psi\right)\right]$,

where the only difference is that the third term in the r.h.s. of eq.(4) has a new exponent $m^{\prime}$ with $m^{\prime}>m$, which can be viewed as $m$ renormalized by the disorder. This result means that the relative strength of the logperiodic correction compared to the first algebraic term becomes smaller as $t$ approaches the time $t_{f}$ of the large earthquake.

However, one must not completely rule out situations where $m^{\prime}<m$. Consider for instance an exponential distribution

$$
p(\lambda)=a e^{-a \lambda} .
$$

Then

$$
<\lambda^{m^{\prime}}>=a^{-m^{\prime}} \Gamma\left(1+m^{\prime}\right),
$$

where $\Gamma$ is the Gamma function. One can then easily show that $m^{\prime}<m$ if both exponents are smaller than $a$, a condition which is realized for sufficiently small values of $\mu$ and large values of $a$. There are even situations where the disorder can completely kill the log-periodic corrections, i.e. only purely real solutions satisfy the pole equation $\left\langle\lambda^{m}\right\rangle=\mu$. The simplest example is the powerlaw

$$
p(\lambda)=\frac{\beta}{\lambda^{1+\beta}}
$$

with $\beta>0$. This leads to

$$
m=\frac{\beta(\mu-1)}{\mu}
$$

as the only solution. The physical interpretation is that the distribution of scale factors from one level to the next of the hierarchical structure is so broad as to destroy the global discrete scale invariance, while recovering an effective global continuous scale invariance at large scales.

We should like to stress that these results hold in an average sense, i.e. after averaging the measured quantities over many disordered configurations. However, when studying specific disordered systems, one expects fluctuations for the $m$ and $m^{\prime}$, from system to system. In fact, we now show that these fluctuations are not selfaveraging when going to large scales (Binder and Young (1986)). To do this, we return to the equation

$$
S_{1}=1+X_{1} S_{2}
$$

and calculate moments relevant to quantify fluctuations. The mean square $\left\langle S_{1}^{2}\right\rangle$ is given by

$$
<S_{1}^{2}>=\frac{1+<X_{1}>}{\left(1-<X_{1}>\right)\left(1-<X_{1}^{2}>\right)},
$$

which is different from $\left\langle S_{1}\right\rangle^{2}$ as soon as $\left\langle X_{1}^{2}\right\rangle \neq<$ $X_{1}>^{2}$. Therefore the variance

$$
\Delta S=<S_{1}^{2}>-<S_{1}>^{2}
$$

is non-vanishing, even in the large scale limit, which concludes the proof of the non-self-averaging property. The variance $\Delta S$ can be written as

$$
\Delta S=\frac{\left.<X_{1}^{2}>-<X_{1}\right\rangle^{2}}{\left(1-<X_{1}>\right)^{2}\left(1-<X_{1}^{2}>\right)} .
$$

Since

$$
<S_{1}>=\frac{1}{1-<X_{1}>},
$$

this shows that the variance also exhibits a singularity for $\left\langle X_{1}\right\rangle=1$, which allows us to write

$$
S_{1}=\frac{1}{1-<X_{1}>}[1+\eta]
$$

where $\eta$ is a random variable of zero mean and variance

$$
\left.<\eta^{2}\right\rangle=\frac{\left\langle X_{1}^{2}>-<X_{1}\right\rangle^{2}}{<X_{1}^{2}>-1} \text {. }
$$

At the singularity, $\left\langle X_{1}\right\rangle=1$, which leads to $\left\langle\eta^{2}\right\rangle=$ 1. This calculation shows that the non-self-averaging property of the observable does not preclude the observation of the singularity which is well-defined at $<$ $X_{1}>=1$; however the relative amplitude of the fluctuations reach $100 \%$ at the critical point, thus providing a possible new signature. 
3 Interplay between dynamics and randomness as a new mechanism of discrete scale invariance

The previous discussion was based on discrete scale invariance, which might appear as a rather strong assumption. We now want to show that such a property is in fact a general consequence of disorder in the dynamics. To make this clear, we consider the simplest model where the divergence of $\dot{\epsilon} \equiv \frac{d \epsilon}{d t}$ is essentially put a priori, and concentrate on the generation of discrete scales. It is possible although lengthy to devise models with more complete features. Let us therefore assume that the seismic release $\dot{\epsilon}$ evolves as a dynamical process with discrete changes. We envision this evolution as a kind of discrete asymmetric random walk with transition rates $w_{+}$for an increase and $w_{-}$for a decrease. The model does not describe the spatial degrees of freedom of the crust but captures its global evolution. The existence of quenched heterogeneity in the crust is captured by assuming that for each value of $\dot{\epsilon}$ corresponds a single couple $\left(w_{+}, w_{-}\right)$of the transition rates. This assumption is fundamental for the generation of an effective discrete scale invariance and the associated log-periodic correction to scaling, as we shall see. More precisely, we assume that for each value $\dot{\epsilon}$,

$$
\begin{cases}\left(w_{+}=u, w_{-}=0\right) & \text { with probability } p \\ \left(w_{+}=u, w_{-}=v\right) & \text { with probability } 1-p\end{cases}
$$

We shall denote the first case a 'diode' situation, since the rate can only increase, while the second case corresponds to a 'two-way' flow. Finally we assume that the evolution occurs itself at a rate

$$
\tau \sim \frac{1}{t_{f}-t}
$$

up to the time of the large event. If $p=1$, this model simply produces the result

$$
\dot{\epsilon} \sim \tau \sim \frac{1}{t_{f}-t} .
$$

We now show that the disorder renormalizes the exponent, and introduces log-periodic corrections.

To do so, assume

$$
\frac{u}{v}<<1-p<<1
$$

The rate increases steadily for the 'diode' $\left(w_{+}=u, w_{-}=\right.$ $0)$ values and gets trapped for some times for the 'twoway' flow cases $\left(w_{+}=u, w_{-}=v\right)$, which, since $\frac{u}{v}<<1$, behave almost as backward diodes. We first claim that this system spontaneously generates a set of discrete 'time' scales. Let us ask what is the typical 'time' $\tau_{k}$ needed for the rate to pass $k$ adjacent 'two-way values'. It is clear that in the limit $\frac{u}{v}<<1$,

$$
\tau_{k} \sim\left(\frac{v}{u}\right)^{k}
$$

Using the fact that the average separation between $m$ tuples is approximately $(1-p)^{-m}$, if $\frac{u}{v}<<(1-p)$, the typical 'time' needed for the energy to go beyond the first $k$-tuple of consecutive 'two-way values' is completely dominated by $\tau_{k}$. One thus expects the rate as a function of 'time' to exhibit local minima at $\tau=\tau_{k}$ : these are the time scales. Moreover the rate increase during the 'time' $\tau_{k}$ is completely dominated by the distance $L$ from the origin to the first k-tuple of consecutive 'two-way' values, that goes like $(1-p)^{-k}$. This derives from the condition

$$
L(1-p)^{k} \sim 1,
$$

writting that the probability $(1-p)^{k}$ for the occurrence of a k-tuple times the total number $L$ of "trials" is the number of k-tuples; then the typical largest $k$ in a $L-$ sequence is such that only one such event occurs among the complete series, hence the condition. In the limit where $\frac{u}{v}<(1-p)<<1$, we can thus write the approximate renormalization group equation

$$
\dot{\epsilon}(\tau) \approx(1-p) \dot{\epsilon}(\lambda \tau)+g(\tau)
$$

where we have set $\lambda \equiv \frac{v}{u}$ and $g(\tau)$ is some regular function taking into account various local effects that correct the main scaling. Because this RG equation can be written only at scales which are powers of $\lambda$, we are back to the discrete scaling invariant case discussed in the first sections. We see in particular that

$$
\dot{\epsilon} \sim \tau^{\alpha}
$$

with

$$
\alpha=1-m=\frac{\log (1-p)}{\log (u / v)} .
$$

Actually, this intuitive argument can be put on a firm footing and has been confirmed in numerical simulations and analytical treatment, carried on in other contexts (Solomon (1975); Bernasconi and Schneiner (1983)), which indeed confirms the existence of log-periodic oscillations in the dependence of the energy as a function of time. The range of parameters over which this holds is much larger than suggested by this intuitive argument. More precisely, as soon as

$$
\frac{u}{v}<(1-p)
$$

one finds

$$
\dot{\epsilon}(\tau) \sim \tau^{\alpha} P\left(\frac{\log \tau}{\log (v / u)}\right)
$$

where

$$
\alpha=\frac{\log (1-p)}{\log (u / v)}
$$

and $P$ is a periodic function of unit period. We thus retrieve the general results obtained from the RG theory, with the correspondance

$$
\lambda=\frac{v}{u}
$$


and

$$
\mu=\lambda(1-p)
$$

For earthquakes, one has $\mu \approx 1.4$ and $\lambda \approx 3$, corresponding to $p \approx .5$ and $u / v \approx 1 / 3$.

We think that this simple model provides a clearer physical meaning to the scaling parameters $\lambda$ and $\mu$ of the renormalization group. We believe that this mechanism for generating log-periodic oscillations, in terms of an interplay between dynamics and quenched randomness, should apply to general situations in a suitable range of parameters corresponding to a regime where the dynamics is highly intermittent.

\section{Non-linear correction to the renormalization group flow : physical consequences}

Up to now, we have mainly discussed the map $\phi(x)$ in the linear approximation around a singularity $x=0$. This corresponds to singling out a large event as the critical point. This seems rather arbitrary and one would like a coherent theory to treat all significant earthquakes on the same footing. Also, in the above picture,large foreshocks are treated as oscillations or corrections, and this is not satisfactory in view of their rather singular character. Remarkably, the same renormalization group formalism, once extended beyond the linear approximation, precisely cures these problems, showing the coherence and aesthetics content of the theory in that it is capable of encompassing a large phenomenology with very few ingredients. Let us get back to the RG equations (1) and (2). So far we have just used local properties of the map $\phi$ by assuming that $x=0$ is an unstable fixed point. However, the global properties of $\phi$ also matter. Quite generally, one can replace the map $\phi$ at next order by

$$
\phi(x) \approx \lambda x-\kappa x^{2},
$$

which includes the leading non-linear term beyond the first linear term expansion around the leading fixed point at $x=0$. Without loosing generality, we can introduce the reduced "time"

$$
y=\frac{\kappa}{\lambda} x
$$

in terms of which eq.(1) transforms into the well known (Collet and Eckmann (1980)) logistic map

$$
y^{\prime}=\lambda y(1-y) \text {. }
$$

Now, if $\lambda$ (whose absolute value is larger than 1) is larger than 2 , the second fixed point of $y^{\prime}(y)$ at $y^{*}=1-\frac{1}{\lambda}$ becomes unstable. Then, this second unstable fixed point is also a critical point which must make $F(x)$ singular. Moreover, it is easy to see ([Derrida et al. (1983)) that $F(x)$ is also singular (i.e. critical) at all the pre-images $\phi^{(-n)}\left(y^{*}\right)$ (for $n=1$ to $+\infty$ ) of $y^{*}$, which accumulate geometrically towards $x=0$, and therefore are equally spaced on a logarithmic scale. The singularities at these preimages correspond precisely to the extrema of the log-periodic term of the linear approximation. Now we see that the main event and its forechocks are treated on a similar basis.

We propose that, beyond the log-periodic corrections, the map $\phi$ itself should be considered as the signature of seismic events. A priori, one can observe a variety of interesting behaviours, including multiple unstable fixed points, unstable periodic orbits and their cortege of associated singularities. One could even expect that the RG flow mapping becomes chaotic, which occurs for $\lambda \geq 3.5699 \ldots$ for the logistic map. In such a case infinitely many periodic orbits become unstable, and the observable $F(x)$ becomes singular at all times (with of course singularities of widly varying strengths), reflecting mathematically the almost permanent occurrence of earthquakes of all sizes.

\section{Conclusion}

In conclusion we believe that the log-periodic corrections, in addition to being unavoidable signatures of discrete scale invariance and very useful in the analysis of data (Sornette and Sammis (1995)), hint at a more complete and global description of seismic activity in terms of the renormalization group transformation $\phi$. Indeed, our theory in terms of $\phi$ describes both the very specific nature of a given earthquake and also its delicate relationship with other earthquakes which concur together to construct the global evolution of the crust. The function $\phi$ could thus provide a novel and more precise quantification tool for earthquakes, their foreshocks and aftershocks.

Acknowledgements. H.S. was supported by the Packard foundation, the National Young Investigator program (NSF-PHY-9357207) and the DOE (DE-FG03-ER40168). C.G.S. was supported by the NSF grant EAR-9304388 and the US Geological survey grant 1434-94-G-2438. D.S. was partially supported by the CNRS-NSF International Cooperation program.

\section{References}

Allègre J.C., J.L. Le Mouel and A. Provost, Scaling rules in rock fracture and possible implications for earthquake prediction, Nature, 297, 47-49, 1982.

Amit D.J., Field theory, the renormalization group and critical phenomena, World Scientific, Singapore, 1984.

Bernasconi J. and W.R. Schneider, Diffusion on a one-dimensional lattice with random asymmetric transition rates, J.Phys.A, 15, I.729-L734, 1983.

Bessis D., J.S. Geronimo and P. Moussa, Complex spectral dimensionality on fractal structures, J.Physique-LETTRES, 44 , L977-[.982, 1.983 .

Binder K. and A.P. Young, Spin-glasses : experimental facts, theoretical concepts and open questions, Rev.Mod.Phys., 58, 801 1986. 
Chelidze T.L., Phys. Earth Planet.Int., 28, 93-101,1982.

Collet P. and J.P. Eckmann, Iterated maps on the interval as dynamical systems, Birkhauser, Boston, 1980.

Cowie P., C.Vanneste and D.Sornette, Statistical physics model for the spatio-temporal evolution of faults, J.Geophys.Res., 98, 21809-21821, 1993.

Derrida B., J.-P. Eckmann and A. Erzan, Renormalization group trajectories with periodic and aperiodic orbits, J.Phys.A, 16, 893-906, 1983.

Jona-Lasinio G., The renormalization group: a probabilistic view, Nuovo Cimento, 26B, 99, 1975.

Keilis-Borok V.I., Introduction : Non-linear systems in the problem of earthquake prediction, Physics of the Earth and Planetary Interiors, 61, 1-7 1990.

Miltenberger P., D. Sornette and C. Vanneste, Fault selforganization as optimal random paths selected by critical spatio-temporal dynamics of earthquakes, Phys.Rev.Lett., 71, 3604-3607, 1993.

Nauenberg M., Scaling representation for critical phenomena, J.Phys. A, 8, 925, 1975.

Newman W., A. Gabrielov, T. Durand, S. L. Phoenix, D.Turcotte, An exact renormalization model for earthquakes and material failure : statics and dynamics, Physica,D77, 200-216, 1994.

Niemeijer Th. and J.M.J. van Leeuwen, in Phase transitions and critical phenomena, Vol.6, C. Domb and M.S. Green, eds. (Academic Press, London, 1976), p.425.

Scholz C., The mechanics of earthquakes and faulting, Cambridge University Press, Cambridge, 1990.

Smalley R.F., Turcotte D.L. and Solla S.A., A renormalization group approach to the strike-slip behavior faults, J.Geophys.Res., 90, 1894, 1985.

Solomon F, Random walks in a random environment, Ann. Prob., $3,1,1975$.

Sornette A. and D. Sornette, Earth rupture as aa criticala point: consequences for telluric precursors, Tectonophysics, 179, 327$334,1990$.

Sornettc D., P. Miltenberger and C.Vanneste, Statistical physics of fault patterns self-organized by repeated earthquakes, $P$ ageop $h$, 142, 491-527, 1994.

Sornette D. and C.G. Sammis, Complex critical exponents from renormalization group theory of earthquakes: Implications for earthquake predictions J.Phys.I France, 5, 607-619, 1995.

Toulouse G. and P. Pfeuly, Introduction au groupe de renormalization et à ses applications, Presse Université de Grenoble, 1975.

Turnarkin A.G. and M.G. Shnirman, Computational seismology, 25, 63-71, 1992.

Wallace D.J. and R.K.P. Zia, Gradient properties of the renormalization group equalions in multicomponent systems, Annals of Physics, 92, 142-163, 1975.

Wilson K.G., Problems of Physics with many scales of length, Scientific American, August 1979, p.140-157. 